\title{
La interpretación sobre el juez nacional en el Tribunal de Justicia de la Comunidad Andina
}

\author{
Yolanda Mendoza*
}

\section{RESUMEN}

Aunque la interpretación prejudicial es considerada como un proceso comunitario no contencioso, sus efectos la convierten en el principal instrumento que el Tribunal de Justicia de la Comunidad Andina emplea con el propósito de garantizar el respeto del derecho comunitario andino. Para cumplir con tan importante labor, el órgano jurisdiccional comunitario necesita de la cooperación de los jueces nacionales, ya que, son ellos los únicos facultados de solicitarle la interpretación prejudicial y de adoptar, en sus decisiones finales, la interpretación que este Tribunal de Justicia realice sobre las normas comunitarias andinas.

En vista de ello, resulta imprescindible contar con una apropiada interpretación del término juez nacional, el mismo que se encuentra descrito en el Artículo 33 del Tratado de Creación del Tribunal de Justicia de la Comunidad Andina. Tal interpretación aseguraría la plena identificación del legítimo operador nacional, considerado también como un juez comunitario por colaborar en la aplicación y en la interpretación uniforme del ordenamiento comunitario andino en el territorio de los Países Miembros de la Comunidad Andina.

Tras analizar las principales sentencias del Tribunal de Justicia de la Comunidad Andina referentes a la interpretación del término juez nacional, verificamos que dicho órgano comunitario, expresamente, no utiliza las reglas de interpretación establecidas en la Convención de Viena sobre el Derecho de los Tratados de 1969. El Tribunal de Justicia opta por una interpretación que amplía su significado y, en consecuencia, entiende que juez nacional también es un funcionario administrativo con función jurisdiccional y un árbitro.

Palabras clave: Comunidad Andina, Tribunal de Justicia de la Comunidad, Derecho Comunitario Andino, Interpretación Prejudicial, Cuestión Prejudicial, Juez Nacional.

Abogada por la Universidad de Lima. Correo electrónico: a20183609@pucp.edu.pe

iD https://orcid.org/0000-0002-3025-5018 
The interpretation on the national judge in the Andean Court of Justice

\section{Abstract}

Although the preliminary interpretation is considered as a non-contentious community process, its effects make it the main instrument used by the Andean Court of Justice with the purpose of guarantee respect for the Andean Community Law. To comply with such important work, the Community court needs the cooperation of national judges, since they are the only ones authorized to request the preliminary interpretation and to adopt, in their final decisions, the interpretation that this Court of Justice makes on the Andean community standards.

In view of that, it is imperative to have an appropriate interpretation of the term national judge, the same one that is described in Article $33^{\circ}$ of the Treaty of Creation of the Andean Court of Justice. Such interpretation would ensure the full identification of the legitimate national operator, also considered as a Community judge for their collaboration in the application and in the uniform interpretation of the Andean legal system in the territory of the Andean Community's Member Countries.

After analyzing the main judgments of the Andean Court of Justice related to the interpretation of the term national judge, we verify that this Community body, expressly, does not use the rules of interpretation established in the Vienna Convention on the Law of Treaties of 1969. This Court of Justice opts for an interpretation that extends its meaning and, consequently, understands that the national judge is also an administrative functionary with a jurisdictional function and an arbitrator.

Keywords: Andean Community, Andean Court of Justice, Andean Community Law, Preliminary Interpretation, Preliminary Ruling, National Judge.

\section{Introducción}

El derecho comunitario andino es el ordenamiento jurídico que regula el proceso de integración de la Comunidad Andina para que esta alcance los objetivos y fines señalados en su tratado constitutivo (el Acuerdo de Integración Subregional o Acuerdo de Cartagena de 1969) como son el de promover el desarrollo equilibrado y armónico de los Países Miembros de la Comunidad Andina a través de la integración y la cooperación económico y social, y el de facilitar su participación en la construcción gradual de un Mercado Común Latinoamericano. El Tribunal de Justicia es el órgano jurisdiccional de la Comunidad Andina, el cual tiene el importante deber de declarar el Derecho Comunitario Andino, y de asegurar su aplicación e interpretación uniforme en todo el territorio de sus Países Miembros.

Desde 2010, el Tribunal de Justicia de la Comunidad Andina interpreta que tanto el árbitro, el juez y el funcionario administrativo que ejerce función jurisdiccional son jueces nacionales de los Países Miembros de la Comunidad Andina, los únicos 
facultados por su tratado de creación de iniciar el proceso comunitario de interpretación prejudicial. Esta controvertida interpretación es el punto de partida del presente artículo, en donde se analizarán los métodos y las reglas de interpretación que tal órgano jurisdiccional utiliza y si las mismas están conforme a lo establecido en la Convención de Viena sobre el Derecho de los Tratados de 1969.

\section{Sobre el proceso de integración andino}

En 1955, la Comisión Económica para América Latina analizó el grado de desarrollo económico de todos los países latinoamericanos y caribeños y recomendó sustituir las importaciones en la región mediante el aumento de la inversión privada y pública dentro de un mercado común regional (Gándara y Bodenstein, 2006, p. 276). Con un enfoque distinto al propuesto en el estudio de esta comisión, los Estados de América Latina y el Caribe elaboraron proyectos e iniciativas de integración de corto alcance territorial cuyas estructuras y fines fueron muy similares a las de las Comunidades Europeas, hoy Unión Europea (Levi, 2006, p. 115).

De esta forma, se suscribieron acuerdos cuyos objetivos estaban referidos al establecimiento de: una Zona de Libre Comercio en América Latina y el Caribe (con el Tratado que instituye la Asociación Latinoamericana de Libre Comercio o Tratado de Montevideo de 1960), un Mercado Común Centroamericano (mediante el Tratado General de Integración Económica Centroamericana de 1960) y una Comunidad Económica de Libre Comercio Caribeña (a través del Acuerdo que establece la Asociación del Libre Comercio del Caribe de 1965).

A causa de las discrepancias internas de la Asociación Latinoamericana de Libre Comercio (Peña y Cárdenas, 1968, p. 11), predecesora de la Asociación Latinoamericana de Integración - en especial sobre la carencia de un trato especial para países con menor desarrollo económico y el incumplimiento de los plazos para establecer una zona de libre comercio- en 1966 un grupo de países de la región andina (conformado por Chile, Colombia, Ecuador, Perú y Venezuela, Bolivia se unió en 1967) decidió acelerar la integración latinoamericana mediante el perfeccionamiento de la Asociación Latinoamericana de Libre Comercio y la posterior unión de esta última con el Mercado Común Centroamericano. Teniendo en cuenta los compromisos jurídicos del Tratado de Montevideo de 1960, este conjunto de países bajo la denominación de Grupo Andino desarrolló un nuevo diseño subregional cuyo proceso de integración se consideraría como una etapa intermedia en la marcha hacia la formación de un Mercado Común Latinoamericano. Esta innovadora fórmula de integración quedó plasmada en el Acuerdo de Integración Subregional (o Acuerdo de Cartagena, en adelante el Acuerdo) del 26 de mayo de 1969 que crea el proceso de integración subregional (en adelante proceso andino). 
Debido a la coyuntura neoliberal que atravesaba la región y frente al incumplimiento constante y progresivo de los objetivos y fines del Acuerdo, mediante el Protocolo Modificatorio del Acuerdo de Integración Subregional Andino de 1996 (conocido como Protocolo de Trujillo) el proceso andino fue reformulado. Este instrumento internacional crea la Comunidad Andina que está conformada por los Países Miembros (Bolivia, Colombia, Ecuador y Perú) y el Sistema Andino de Integración (Artículo 5 del Acuerdo). Bajo esta nueva denominación, el proceso andino es el único que cuenta con un ordenamiento jurídico comunitario como tal, es decir, caracterizado por los principios de primacía, de aplicabilidad directa y efecto directo en la región. Asimismo, el proceso andino se encuentra asistido y promovido por los órganos e instituciones del Sistema Andino de Integración (SAI) de la Comunidad Andina (Artículo 7 del Acuerdo), entre los que se encuentra el Tribunal de Justicia de la Comunidad (en adelante el Tribunal Andino), que es el órgano jurisdiccional de la Comunidad Andina (Artículo 40 del Acuerdo).

Tal corriente reformadora del proceso andino, también alcanzó al funcionamiento y las competencias de ciertos órganos comunitarios del SAI, como el Tribunal Andino. Originariamente, la estructura del proceso andino carecía de órgano comunitario capaz de garantizar la legalidad y el respeto del Acuerdo, así como demás actos derivados del mismo. Por aquel entonces, cualquier tipo de controversias, en especial las referidas a la interpretación o la ejecución del Acuerdo, eran resueltas por la Comisión mediante la negociación, buenos oficios, mediación y conciliación (Artículo 23 de la versión original del Acuerdo de Cartagena). Además, agotados tales medios los Países Miembros del Acuerdo debían utilizar los procedimientos establecidos en el Protocolo para la Solución de Controversias de 1967, el mismo que era parte del ordenamiento jurídico de la Asociación Latinoamericana de Libre Comercio.

Dicha carencia fue subsanada con la suscripción del Tratado que crea el Tribunal de Justicia del Acuerdo de Cartagena del 28 de mayo de 1979 (en adelante el Tratado) y que fue modificado por el Protocolo Modificatorio del Tratado de Creación del Tribunal de Justicia del Acuerdo de Cartagena del 28 de mayo de 1996. Con este tratado se crea el Tribunal Andino como órgano jurisdiccional de la Comunidad Andina, con sede en Quito, y regido por el tratado y sus protocolos modificatorios (Artículo 5 del Tratado), y por el Acuerdo (Artículo 41 del Acuerdo).

Asimismo, el funcionamiento del Tribunal Andino y sus procedimientos, además del desarrollo de las normas del tratado, se encuentran regulados por el Estatuto del Tribunal Andino (en adelante el Estatuto, recogido en la Decisión 500 del Consejo Andino de Ministros de Relaciones Exteriores del 22 de junio de 2001), y por el Reglamento Interno del Tribunal Andino del 18 de mayo de 2004. De otro lado, 
un punto importante del tratado (Artículo 1) es su exposición de cómo el Derecho Comunitario Andino, es decir el ordenamiento jurídico comunitario andino (en adelante el ordenamiento andino) está conformado; además, el Estatuto (Artículo 2) señala cuáles son las fuentes directas del ordenamiento andino:

i. las normas originarias: el Acuerdo, sus Protocolos e Instrumentos adicionales; y el Tratado y sus Protocolos modificatorios que crea el Tribunal Andino.

ii. las normas derivadas: las Decisiones del Consejo Andino de Ministros de Relaciones Exteriores y de la Comisión de la Comunidad Andina; las Resoluciones de la Secretaría General de la Comunidad Andina; y los Convenios de Complementación Industrial y otros que adopten los Países Miembros entre sí en el marco del proceso de integración subregional andino.

Una parte de la doctrina difiere en esta clasificación y propone la existencia de una fuente complementaria en este ordenamiento, conformada por los Convenios de Complementación Industrial (Novak, 2003, pp. 61 y 62). Otra parte de la doctrina, conforme a la apreciación del Tribunal Andino, considera la presencia de fuentes indirectas tales como la jurisprudencia del Tribunal Andino, la costumbre y los principios generales del Derecho, siempre y cuando sean comunes en los ordenamientos internos de los Países Miembros de la Comunidad Andina (Quindimil, 2006, p. 173).

\section{La interpretación prejudicial en la Comunidad Andina}

\subsection{Objetivo de la interpretación prejudicial}

Como órgano jurisdiccional supranacional que declara el derecho comunitario andino y garantiza la aplicación e interpretación uniforme del mismo en todo el territorio de los Países Miembros (Artículo 4 del Estatuto), el Tribunal Andino es competente para llevar a cabo la interpretación prejudicial. A través de ella, el órgano jurisdiccional de la Comunidad Andina se encuentra facultado para interpretar las normas comunitarias andinas, aunque estas hayan sido adoptadas en los ordenamientos internos de los Países Miembros, para así asegurar la aplicación uniforme del ordenamiento andino en todo el territorio de los Países Miembros de la Comunidad Andina (Artículo 32 del Tratado). Lo último fue establecido por el Tribunal Andino en su sentencia del 3 de diciembre de 1987 referente al «Proceso $\mathrm{N}^{\circ}$ 1-IP-87: Interpretación prejudicial de los artículos 58, 62 y 64 de la Decisión 85 de la Comisión del Acuerdo de Cartagena, solicitada por el Consejo de Estado de la República de Colombia».

Si bien la interpretación prejudicial es un proceso comunitario no contencioso, es también una herramienta fundamental que coadyuva con el cumplimiento de los objetivos y fines del proceso andino. Al garantizarse dicha aplicación uniforme se propicia el logro del bienestar e interés común de los habitantes de la Comunidad 
Andina (Artículo 1 del Acuerdo). Asimismo, la interpretación prejudicial es entendida como un mecanismo de cooperación y consulta entre los jueces nacionales y el Tribunal Andino (Perotti, 2002, p. 130), con el objetivo principal de aplicar uniformemente el ordenamiento andino. Dicho de otro modo, no existe una relación de jerarquía y, en consecuencia de subordinación, entre los jueces nacionales y los magistrados del Tribunal Andino, ya que ambos cumplen el rol de juez comunitario a fin de encontrar el adecuado sentido del ordenamiento andino que, según sus características mencionadas, ya se encuentra implementado dentro de los ordenamientos internos de los Países Miembros de la Comunidad Andina sin necesidad de tomar medidas de interiorización.

\subsection{Requisitos para plantear la interpretación prejudicial}

La interpretación prejudicial se inicia por solicitud de los jueces nacionales de los Países Miembros de la Comunidad Andina (Artículo 33 del Tratado), siempre y cuando en algún proceso de sus jurisdicciones internas exista la obligación de aplicar alguna norma comunitaria andina cuya interpretación sea decisiva para la resolución de la controversia interna (Novak, 2003, p. 96). Esta solicitud podrá ser promovida de oficio o a petición de parte (Artículo 33 del Tratado) y será presentada por el juez nacional al Tribunal Andino en cualquier momento del proceso interno antes de dictar sentencia.

Además, la solicitud de interpretación prejudicial es facultativa si la resolución del proceso nacional puede ser impugnada por algún recurso interno, o de lo contrario será obligatoria, si la interpretación prejudicial es solicitada por el juez nacional de única o última instancia con la respectiva suspensión del proceso nacional hasta recibir la interpretación del Tribunal Andino. Sea cual fuere el caso, toda solicitud de interpretación prejudicial que el juez nacional dirija al Tribunal Andino deberá contener el nombre e instancia del juez o tribunal nacional consultante, la relación de las normas del ordenamiento andino cuya interpretación se requiere, la identificación de la causa que origine la solicitud, el informe sucinto de los hechos que el solicitante considere relevantes para la interpretación, y el lugar y la dirección en que el juez nacional recibirá la respuesta a su consulta (Artículo 125 del Estatuto).

En el caso que la solicitud de interpretación prejudicial sea admitida, el Tribunal Andino cuenta con treinta días para pronunciarse y dictar sentencia (Artículo 126 del Estatuto), y solo se limitará a precisar el contenido y alcance de las normas comunitarias andinas cuya interpretación se ha solicitado (Artículo 34 del Tratado). El resultado del proceso interpretativo del Tribunal Andino, que implica un ejercicio intelectual por el que se investiga cuál es el significado de una disposición (Kolb, 2016, p. 128), deberá de ser adoptado por el juez nacional solicitante en su sentencia 
(Artículo 35 del Tratado). En caso que el juez nacional aplique una interpretación distinta a aquella establecida por el órgano jurisdiccional comunitario, los Países Miembros de la Comunidad Andina y los particulares que se vean afectados por esta decisión, podrán iniciar la 《acción de incumplimiento》 ante el Tribunal Andino (Artículo 128 del Estatuto).

\subsection{Principales efectos jurídicos de la interpretación prejudicial}

Uno de los principales efectos jurídicos de este recurso es la fuerza vinculante para el juez nacional que solicitó la interpretación prejudicial, ya que tendrá que fundamentar su resolución en base a la interpretación del Tribunal Andino y ajustar sus decisiones finales a la misma (Quindimil, 2006, p. 413).

Cabe señalar que, el órgano jurisdiccional de la Comunidad Andina no considera sus propias resoluciones de interpretaciones prejudiciales como precedentes, sino que interpreta "para cada caso concreto». El órgano jurisdiccional andino justifica esta decisión con el objetivo de activar y, posteriormente, consolidar el Derecho Comunitario Andino. Así lo refiere en la sentencia del 7 de agosto de 1995 sobre el «Proceso 04-IP-94: Solicitud de Interpretación prejudicial de los artículos 58, literal f), 66, 67 y 72 de la Decisión 85 de la Comisión del Acuerdo de Cartagena, formulada por el Consejo de Estado de la República de Colombia». En vista de lo dispuesto por el Tribunal Andino, los jueces nacionales tendrán que realizar las consultas aun cuando la norma comunitaria andina les sea clara o entendible.

Otro de los efectos se refiere a que la interpretación prejudicial sirve como un mecanismo de protección que los particulares pueden utilizar, en el sentido que ellos pueden advertir al juez nacional de la existencia de normas comunitarias en el proceso interno, a fin de proteger los derechos que el ordenamiento andino les reconoce.

Finalmente, la interpretación prejudicial permite que el ordenamiento andino se aplique de manera uniforme, se desarrolle y consolide; la estabilidad del mismo dependerá de la frecuencia de las solicitudes de interpretación prejudicial y de la calidad de las resoluciones que pueda emitir el Tribunal Andino.

\section{El término «juez nacional» quien solicita la interpretación prejudicial}

Como hemos explicado, los jueces nacionales de los Países Miembros de la Comunidad Andina son los únicos que tienen la capacidad de solicitar, directamente al Tribunal Andino, la interpretación prejudicial a fin de resolver las controversias que se les han asignado y en donde se discuta alguna norma del ordenamiento andino. Desde que el Tribunal Andino inició sus labores en 1984, el significado del término «juez 
nacional» del Artículo 33 del Tratado de Creación del Tribunal de la Comunidad Andina ha sido entendido o interpretado por este, de diversas formas.

En efecto, sabemos que no existe una forma de interpretar un tratado, debido a que frente a un significado ambiguo pueden surgir distintas formas de descifrar los tratados (Linderfalk, 2010, p. 10). Esto fue advertido en 1949 por la Comisión de Derecho Internacional de la Organización de las Naciones Unidas, entendiendo la interpretación como un arte y no una ciencia (Kolb, 2016, p. 134). En vista de ello, se decidió sintetizar tanto las reglas básicas o consuetudinarias de interpretación de los tratados (Cannizzaro, 2011, p. 118) como el orden de prelación que debería ser utilizado durante el proceso interpretativo (Linderfalk, 2010, p. 3) en los Artículos 31 y 32 de la Convención de Viena sobre el Derecho de los Tratados de 1969 (en adelante la Convención).

Denominada como la guía en la interpretación de los tratados (Orakhelashvili, 2008, p. 313) para los Estados, los órganos jurisdiccionales internacionales, los órganos estatales, los órganos de una Organización Internacional y los publicistas, todos ellos facultados de interpretar (Novak, 2013, pp. 72-73), la Convención recoge la interpretación textual, sistemática, teleológica o funcional como reglas básicas de interpretación (Linderfalk, 2010, p. 8). De igual modo, recoge ciertos medios de interpretación que se utilizarán como complementarios (Artículo 32 de la Convención), siempre y cuando tras la aplicación de las reglas generales de interpretación (Artículo 31 de la Convención) no se haya logrado comprender el sentido ordinario del tratado (Villiger, 2009, p. 446) y persista la ambigüedad o un resultado absurdo o irrazonable.

En el caso de la Comunidad Andina, es necesario recalcar que los Países Miembros delegaron al Tribunal Andino la facultad de interpretar el ordenamiento andino. Durante los primeros años de su funcionamiento, el órgano jurisdiccional comunitario empleó la interpretación textual para comprender el ordenamiento andino. Sin embargo, además de expresar la utilización de la interpretación teleológica o funcional en la ya mencionada sentencia del 3 de diciembre de 1987, el Tribunal Andino aclaró que no dejaría de emplear los demás métodos universalmente admitidos para interpretar correctamente el ordenamiento andino (Novak, 2003, p. 98).

A continuación desarrollaremos los alcances del término juez nacional pues es quien solicita la interpretación prejudicial realizada por el Tribunal Andino.

\subsection{Como juez ordinario}

Inicialmente, conforme a lo desarrollado en el «Proceso 1-89 sobre la consulta formulada por la doctora Angela Vivas Martínez», el Tribunal Andino reconocía que 
solo los jueces nacionales de los Países Miembros de la Comunidad Andina eran los únicos que poseían la facultad de solicitar la interpretación prejudicial tal y como lo disponía el Artículo 29 del Tratado que crea el Tribunal de Justicia del Acuerdo de Cartagena. De otro lado, resulta necesario señalar que en el sexto párrafo de su providencia del 25 de abril de 1989 respecto del Proceso 1-89, el Tribunal Andino no fundamentó correctamente los motivos de su interpretación; solo considera que los particulares no pueden solicitarle directamente la interpretación prejudicial. Más bien, del análisis de esta providencia se desprende que el Tribunal Andino utilizó la interpretación textual, la misma que es considerada como el inicio del proceso interpretativo (Villiger, 2009, p. 426) y que arroja un número de significados ordinarios dependiendo de las circunstancias y época en que la interpretación se realice (Cannizzaro, 2011, p. 109). En ningún momento, el Tribunal Andino mencionó las reglas de interpretación de la Convención, a pesar que justifica su decisión en las disposiciones de su tratado de creación.

Realizando una comparación del derecho comunitario andino con el ordenamiento jurídico de la Unión Europea, el Artículo 267 del Tratado de Funcionamiento de la Unión Europea indica que el órgano jurisdiccional nacional de uno de los Estados Miembros es el encargado de solicitar la cuestión prejudicial al Tribunal de Justicia de la Unión Europea sobre la interpretación de sus tratados, y respecto a la validez e interpretación de los demás actos derivados del mismo. Como vemos, la redacción de este Artículo 267 es muy diferente al del Artículo 33 del tratado de creación del tribunal de la Comunidad Andina ya que, no es el juez nacional (sujeto que ejerce función judicial) de algún Estado Miembro de la Unión Europea el encargado de remitir la cuestión prejudicial sino algún órgano jurisdiccional nacional. No obstante, la única similitud entre los artículos de interpretación prejudicial y cuestión prejudicial que podría ser considerada refiere a que ninguno define el término juez nacional ni el de órgano jurisdiccional, respectivamente.

Tras un examen de la aplicación del derecho de la Unión Europea y en caso el órgano jurisdiccional de algún Estado Miembro tenga dudas razonables sobre el entendimiento de las normas de la Unión Europea que deban ser utilizadas para resolver los litigios que están a su cargo, dicho órgano jurisdiccional tanto facultativa como obligatoriamente remitirá la cuestión prejudicial al Tribunal de Justicia, para que tal institución se pronuncie a fin de resolver correctamente la controversia que le ha sido asignada conforme al ordenamiento nacional. En cuanto a su labor interpretativa, debido a la autonomía de este derecho sui generis frente al derecho internacional, desde los inicios de su funcionamiento el Tribunal de Justicia de la Unión Europea utiliza el método teleológico para interpretar sus Tratados Fundacionales y 
Modificatorios, dado que los mismos indican una serie de objetivos y fines para la consolidación de la integración europea (Del Valle, 1995, p. 863).

Con la sentencia del Tribunal de Justicia de la Unión Europea del 30 de junio de 1966 sobre el «Asunto 61/65 Veuve G. Vaassen-Göbbels contra Bestuur van Beambtenfonds voor het Mijnbedrijf. Petición de decisión prejudicial: Scheidsgerecht van het Beambtenfonds voor het Mijnbedrijf Heerlen - Países Bajos» empieza el camino interpretativo de esta institución referente al término órgano jurisdiccional. En el punto I de la sentencia, este tribunal de justicia señala que su concepto es uno muy distinto de los que pueden proporcionar los ordenamientos jurídicos nacionales de los Estados Miembros de la Unión Europea. Asimismo, en esta sentencia el Tribunal consideró cinco criterios para definir el órgano jurisdiccional legitimado a solicitarle la cuestión prejudicial: el origen legal, la permanencia, el principio de contradicción, el carácter obligatorio y la aplicación de normas del Derecho de la Unión Europea. En cuanto al método de interpretación que se utilizó para determinar tales criterios, el escogido por el Tribunal de Justicia de la Unión Europea fue el teleológico ya que, como es explicado en el párrafo anterior, es considerado el más adecuado para interpretar las disposiciones de un cuerpo normativo como el europeo.

Posteriormente, la sentencia del Tribunal de Justicia (Sala Quinta) del 11 de junio de 1987 sobre el «Asunto 14/86 Pretore di Salò contra X. Petición de decisión prejudicial: Pretura di Salò - Italia. Prejudicial - Agresiones al medio ambiente» agrega un criterio más para definir el órgano jurisdiccional de los Estados Miembros. En el numeral 7 de su sentencia, el Tribunal de Justicia de la Unión Europea señala a «la independencia del órgano jurisdiccional para juzgar conforme a Derecho aunque no tenga un carácter estrictamente jurisdiccional» como el sexto criterio a valorar y que todo órgano jurisdiccional nacional que plantee la cuestión prejudicial deberá demostrar. Téngase en cuenta que, para el Tribunal de Justicia también es muy importante que el órgano jurisdiccional encargado de remitirle la cuestión prejudicial resuelva un conflicto jurídico, es decir un litigio. Lo último, fue indicado en el numeral 4 del Auto del Tribunal de Justicia del 5 de marzo de 1986 sobre el «Asunto 318/85 Procedimento penal entablado contra Regina Greis Unterweger. Petición de decisión prejudicial: Commissione consultiva per le infrazioni valutarie - Italia. Incompetencia del Tribunal».

\subsection{Como funcionario administrativo con función jurisdiccional}

En 1987, el Tribunal Andino consideró por primera vez que los funcionarios administrativos de los Países Miembros de la Comunidad Andina que cumplen funciones jurisdiccionales también ejercen las mismas facultades que un juez nacional. Así lo estableció en la señalada sentencia del 3 de diciembre de 1987 sobre el «Proceso Nº 1-IP-87 
Interpretación prejudicial de los artículos 58, 62 y 64 de la Decisión 85 de la Comisión del Acuerdo de Cartagena, solicitada por el Consejo de Estado de la República de Colombia», conocido como el Caso Sociedad Aktiebolaget Volvo. En el tercer visto de su sentencia, el Tribunal Andino señaló que el Consejo de Estado de la República de Colombia, al ser máxima instancia de la justicia contenciosa administrativa colombiana, actuaba como un tribunal nacional en cumplimiento de una función judicial.

Por tanto, si el Consejo de Estado de la República de Colombia era entendido como una instancia jurisdiccional nacional, sus funcionarios administrativos también serían los equivalentes de los jueces nacionales. De otro lado, en el punto 3.5 de la mencionada sentencia, el Tribunal Andino mencionó que utilizó la interpretación teleológica o funcional, la misma que indaga y aclara cuál es la funcionalidad del tratado (Villiger, 2009, p. 426) para interpretar el término juez nacional. A pesar que en la sentencia de este caso, el Tribunal Andino afirmó que la interpretación teleológica o funcional se adapta mejor para entender el objeto y fin del Acuerdo, no hizo referencia expresa a las reglas de interpretación de la Convención.

En 2007, con el «Proceso 14-IP-2007 Interpretación prejudicial de los artículos 136, literales a) y b), 155, literales a), c) y d), 157, párrafo primero, 258, 259, párrafo primero y literal a), y 267 de la Decisión 486 de la Comisión de la Comunidad Andina, y de oficio, de los artículos 238 y 268 de la mencionada Decisión, así como, el artículo 33 del Tratado de Creación del Tribunal de Justicia de la Comunidad Andina, con fundamento en la consulta formulada por la Superintendencia de Industria y Comercio, República de Colombia, Grupo de Trabajo de Competencia Desleal», denominado Caso Industrias Mayka S.A., el Tribunal Andino aclaró que es lo que entendía por el término juez nacional. En la Consideración A del punto VI de su sentencia del 21 de marzo de 2007, el órgano jurisdiccional de la Comunidad Andina explicó que los actos judiciales no solo son los que emanan del Poder Judicial sino también de otros órganos que los Países Miembros de la Comunidad Andina les hayan otorgado función judicial, conforme a las disposiciones mínimas legales de su ordenamiento nacional. Así pues, para el órgano jurisdiccional andino cualquier sujeto que ostenta función judicial de algún País Miembro de la Comunidad Andina tendrá que ser considerado como juez nacional y poseerá la capacidad y la legitimidad de solicitarle la interpretación prejudicial.

Sin más fundamento que el de indicar que realiza una interpretación amplia del término juez nacional a fin identificar quién es el sujeto con capacidad de iniciar este procedimiento no contencioso y a pesar de invocar el Artículo 33 del Tratado, el Tribunal Andino mantuvo su postura de no realizar ninguna alusión a las reglas de interpretación de la Convención. Asimismo, recalcó que su proceso interpretativo está en concordancia con la finalidad de la interpretación prejudicial y que es 
la aplicación uniforme del ordenamiento andino en todo el territorio de los Países Miembros de la Comunidad Andina. Como vemos, el órgano jurisdiccional comunitario andino persistió con su labor interpretativa de tipo teleológica o funcional, sin hacer referencia a los métodos de interpretación recogidos tanto en la Convención como los señalados en la doctrina; en este último caso, por ejemplo, el principio de contemporaneidad y la regla de la intertemporalidad (Novak, 2013, pp. 81-86).

En ese mismo año, el Tribunal Andino a través del «Proceso 130-IP-2007: Interpretación prejudicial de los artículos 221, 222, 223, 258, 259 literal c) y 267 de la Decisión 486 de la Comisión de la Comunidad Andina formulada por la Superintendencia de Industria y Comercio de la República de Colombia. Grupo de Trabajo de Competencia Desleal. Interpretación, de oficio, de los artículos 33 del Tratado de Creación del Tribunal de Justicia de la Comunidad Andina, 238, 241, 243, 268 y 269 de la Decisión 486 de la Comisión de la Comunidad Andina», llamado Caso Yanbal de Colombia S.A., reafirmó el uso de la interpretación amplia del término juez nacional del Artículo 33 del Tratado. En su sentencia del 17 de octubre 2007, el Tribunal Andino reiteró íntegramente el fundamento que utilizó para resolver el Proceso 14-IP-2007 y recalcó, en la Consideración 3 de dicha sentencia, la importancia del principio de cooperación y colaboración entre el juez nacional y el juez comunitario.

Hace casi cinco años, a través del «Proceso 121-IP-2014: Interpretación prejudicial del artículo 237 de la Decisión 486 de la Comisión de la Comunidad Andina y, de oficio, de los artículos 273 de la Decisión 486 y 122 del Estatuto del Tribunal de Justicia de la Comunidad Andina», conocido el Caso Marfor Producciones S.R.L contra Exponáutica Perú, el Tribunal Andino estableció, en el punto 29 de su sentencia del 20 de noviembre de 2014, seis criterios generales (muy similares a lo establecido por el Tribunal de Justicia de la Unión Europea en la sentencia del caso Vaassen-Göbbels) que todo órgano administrativo con función jurisdiccional deberá acreditar para calificar como juez nacional capaz de solicitarle la interpretación prejudicial:

i. que sea constituido por mandato legal;

ii. que se trata de un órgano permanente;

iii. el carácter obligatorio de sus competencias;

iv. que tenga la obligación de aplicar el ordenamiento andino;

v. el carácter contradictorio de los procedimientos a su cargo y el debido respeto; y

vi. la imparcialidad de sus actos.

Es necesario agregar que, de acuerdo con lo dispuesto en el punto 17 de la mencionada sentencia, para el Tribunal Andino el término juez nacional es uno autónomo 
y en consecuencia distinto a las definiciones nacionales de los Países Miembros de la Comunidad Andina, inclusive estas podrían atentar contra el derecho del acceso a la justicia comunitaria y restringir la aplicación del ordenamiento andino en todo el territorio de los Países Miembros de la Comunidad Andina. Esta nueva postura del Tribunal Andino también fue tomada de la reiterada jurisprudencia del Tribunal de Justicia la Unión Europea sobre el caso Vaassen-Göbbels de 1966.

Siguiendo el planteamiento de anteriores posiciones respecto de la interpretación prejudicial del término juez nacional, el Tribunal Andino reiteró la justificación realizada en los Procesos 14-IP-2007 y 130-IP-2007 sobre la importancia de la aplicación uniforme del ordenamiento andino en todo el territorio de los Países Miembros de la Comunidad Andina. Hay que mencionar además que, el Tribunal Andino se equivocó en precisar - como así lo señala en el punto 18 de la sentencia del Caso Marfor Producciones S.R.L contra Exponáutica Perú- que hasta ese momento no había realizado una «interpretación restrictiva y mucho menos literal» del término juez nacional, cuando en realidad, en el ya mencionado Proceso 1-89, comprobamos que sí realizó un proceso interpretativo literal o textual de dicho término para declarar la inadmisibilidad de la consulta formulada por la Doctora Angela Vivas Martínez. A pesar de ello, el Tribunal Andino precisa que para entender el término juez nacional tiene en cuenta los objetivos y fines del tratado, los mismos que la han llevado a realizar a través de los años una interpretación amplia del mismo. Obsérvese que, el Tribunal Andino continúa utilizando la interpretación teleológica o funcional para encontrar el significado del término juez nacional y sigue sosteniendo, como su principal fundamentación de su amplia interpretación, que es cualquier sujeto que realice función judicial.

Como prueba que respalda tal método de interpretación, en la citada sentencia del Caso Marfor Producciones S.R.L contra Exponáutica Perú, el órgano jurisdiccional de la Comunidad Andina hace un listado de los principales procedimientos de interpretación prejudicial provenientes de funcionarios administrativos con función jurisdiccional, y que habían sido presentados hasta el 2014. Por ejemplo, menciona los siguientes: el Tribunal Administrativo del Atlántico (Proceso 30-IP98), la Superintendencia de Industria y Comercio de la República de Colombia «Grupo de Trabajo de Competencia Desleal» (Procesos 14-IP-2007 y 130-IP2007), la Corte Constitucional de la República de Colombia (Procesos 10-IP-94, 01-IP-96, 60-IP-2012), la Corte Suprema de Justicia de la República de Venezuela (Proceso 19-IP-98), la Corte Suprema de Justicia de la República del Perú (149-IP2011), el Tribunal Constitucional Plurinacional de Bolivia (Proceso 67-IP-2014), Tribunales Arbitrales de la Cámara de Comercio de Bogotá (Procesos 161-IP-2013, 181-IP-2013, 14-IP-2014), el Centro de Arbitraje de la Cámara de Comercio de Medellín (Proceso 79-IP-2014), y el Centro de Arbitraje de la Cámara de Comercio 
Ecuatoriana-Americana (Proceso 262-IP-2013). A diferencia de sus anteriores motivaciones, en el Proceso 121-IP-2014 el Tribunal Andino mejora los fundamentos y las argumentaciones de su resolución para continuar con el empleo de la interpretación teleológica o funcional. Sin embargo, su proceso interpretativo del significado del término juez nacional sigue mostrando un resultado muy cuestionable.

Reiteramos que el Tribunal Andino sigue la línea argumentativa del Tribunal Justicia de la Unión Europea para analizar si un juez nacional reúne todos los requisitos para que tenga legitimidad de solicitarle la interpretación prejudicial. Por ello, además de guiarse de jurisprudencia del Caso Vaassen-Göbbels, el Tribunal Andino sigue la posición del Tribunal de Justicia de la Unión Europea en lo que se refiere a la verificación de las funciones del juez nacional que solicita la interpretación prejudicial. Así lo podemos constatar con el Auto del Tribunal de Justicia del 26 de noviembre de 1999 referente al «Asunto C-192/98 Azienda nazionale autonoma delle strade (ANAS). Petición de decisión prejudicial: Corte dei Conti - Italia. Artículo 177 del Tratado CE (actualmente, artículo $234 \mathrm{CE}$ ) - Concepto de «órgano jurisdiccional de uno de los Estados miembros» - Directiva 92/50/CEE - Procedimientos de adjudicación de los contratos públicos de servicios». En el numeral 23 del Auto, el Tribunal de Justicia de la Unión Europea señala que aunque el ordenamiento nacional de los Estados Miembros de la Unión Europea otorgue función jurisdiccional a ciertos órganos internos, es necesario que «este Tribunal verifique cuál es la naturaleza específica de las funciones que ejerce en el contexto normativo particular en el que se ve obligado a pedir al Tribunal de Justicia que se pronuncie».

En este contexto, es preciso señalar que el Tribunal de Justicia de la Unión Europea consideró como un órgano jurisdiccional, a los efectos de la remisión de la cuestión prejudicial, también a:

i. órganos jurisdiccionales de los países y territorios de ultramar de los Estados Miembros de la Unión Europea: decisión que empezó con la solicitud del Tribunal administratif de Papeete (Polinesia francesa), como así lo demostró en los numerales 6 al 10 de la sentencia del Tribunal de Justicia de la Unión Europea del 12 de diciembre de 1990 sobre los «Asuntos acumulados C-100/89 y C-101/89: Peter Kaefer y Andréa Procacci contra Estado francés. Peticiones de decisión prejudicial: Tribunal administratif de Papeete (Polinesia) - Francia. Derecho de residencia y de establecimiento - Países y territorios de ultramar Artículo 177 - Competencia del Tribunal de Justicia».

ii. órgano jurisdiccional común a varios Estados miembros: si se presentase el caso en que un órgano jurisdiccional vea la necesidad de interpretar normas del Derecho de la Unión Europea cuya decisión no es susceptible de impugnación a fin de cumplir con su labor por la cual fue creada, dicho órgano si es común a varios Estados Miembros tiene la obligación de reenviar la cuestión prejudicial. 
Así lo estableció el Tribunal de Justicia de la Unión Europea en los numerales 20 al 32 de la sentencia del Tribunal de Justicia sobre el «Asunto C-337/95: Parfums Christian Dior SA y Parfums Christian Dior BV contra Evora BV. Petición de decisión prejudicial: Hoge Raad - Países Bajos. Derecho de marca y derecho de autor - Acción del titular de estos derechos que pretende que se prohíba a un comerciante hacer publicidad para la comercialización ulterior del producto - Perfume», al declarar al Tribunal de Justicia del Benelux como órgano jurisdiccional para los fines de la cuestión prejudicial.

De lo expuesto hasta este punto, consideramos que el Tribunal Andino no evalúa con rigurosidad las facultades propias del funcionario administrativo con función jurisdiccional. Esta decisión técnica del tribunal, conjuntamente con la de crear sus propias definiciones, ocasiona que el órgano jurisdiccional comunitario andino interprete que «juez nacional» también es funcionario administrativo con función jurisdiccional. A nuestro parecer, el análisis inadecuado del Tribunal Andino causa más incoherencias para la seguridad jurídica del ordenamiento andino porque la naturaleza de esas dos figuras es diferente. Si bien el funcionario administrativo con función jurisdiccional puede utilizar medidas coactivas para que los administrados cumplan con sus resoluciones, definitivamente no puede ejercer ninguna medida coercitiva. Esta potestad es consustancial y propia de todo juez nacional, quien además está encargado de controlar las decisiones del funcionario administrativo en un proceso contencioso administrativo.

\subsection{Como árbitro}

En cuanto a considerar al árbitro dentro del significado amplio que tiene el Tribunal Andino del término juez nacional, esta cuestión es revisada en el «Proceso 03-AI2010: Acción de incumplimiento interpuesta por la Empresa de Telecomunicaciones de Bogotá S.A. ESP, (ETB S.A. E.S.P.) contra la República de Colombia, Sección Tercera del Consejo de Estado, por supuesto incumplimiento de la obligación objetiva de solicitar interpretación prejudicial obligatoria prevista en los artículos 4, 33, 35 y 36 del Tratado de Creación del Tribunal de Justicia de la Comunidad Andina y en los artículos 122, 123, 124, 127 y 128 de la Decisión 500, Estatuto del Tribunal de Justicia de la Comunidad Andina», conocido como el Caso Empresa de Telecomunicaciones de Bogotá S. A. ESP. En el considerando 10 de su sentencia del 26 de agosto de 2011, el Tribunal Andino reconoció que directamente los árbitros en derecho cuando actúan en «última instancia ordinaria» tienen la obligación de solicitar la interpretación prejudicial, ya que ellos poseen funciones similares de los jueces nacionales como son la de administrar justicia, la independencia y sus decisiones tienen efectos muy similares al de la cosa juzgada. 
El Tribunal Andino fundamentó esta interpretación por considerar que el Consejo de Estado de la República de Colombia, previamente reconocido como órgano administrativo con función jurisdiccional, debió resolver la nulidad de tres laudos arbitrales (sobre las controversias entre COMCEL - ETB S.A. E.S.P., OCCEL - ETB S.A. E.S.P. y CELCARIBE - ETB S.A. E.S.P.) teniendo en cuenta la correcta aplicación del ordenamiento andino (la Decisión 462 que regula el Proceso de Integración y Liberalización del Comercio de Servicios de Telecomunicaciones) y, en consecuencia, solicitar la correspondiente interpretación prejudicial. Además, señala que realiza una interpretación extensiva o amplia del término juez nacional quien solicita la interpretación prejudicial. Al igual que en los anteriores procesos, el Tribunal Andino tampoco hizo referencia a la utilización de las reglas de interpretación de la Convención.

Siguiendo con la misma línea interpretativa de asemejar al árbitro con el juez nacional, en 2012 el Tribunal Andino resolvió el «Proceso 57-IP-2012: Interpretación prejudicial, de oficio, de los artículos 32 y 33 del Tratado de Creación del Tribunal de Justicia de la Comunidad Andina, y 122 y 123 de la Decisión 500 de 22 de junio de 2001 (Estatuto del Tribunal de Justicia de la Comunidad Andina), con fundamento en la consulta solicitada por la Sección Tercera del Consejo de Estado de la República de Colombia» conocido como el Caso Comcel S.A. Mediante el considerando 4 de su sentencia del 11 de julio de 2012, el Tribunal Andino reiteró la interpretación extensiva o amplia del término juez nacional citando los fundamentos del Proceso 03-AI-2010 y recalcó que «la interpretación prejudicial es esencial, básica y angular para el funcionamiento del sistema de integración subregional». De otro lado, en el considerando 5, el Tribunal Andino afirmó que la omisión de la solicitud obligatoria de la interpretación prejudicial por el juez nacional podría originar una denuncia por incumplimiento del ordenamiento andino contra el País Miembro y la nulidad de la sentencia que emita, así como podría causar «una violación al derecho al debido proceso». Frente a esta última situación, el Tribunal Andino explicó que la parte afectada con tal omisión del juez nacional podría interponer los medios impugnatorios y acciones constitucionales permitidos por su respectivo ordenamiento interno.

Por otra parte, el Tribunal Andino indicó también, en el considerando 5 de la sentencia del Caso Comcel S.A., dos hipótesis que las instancias judiciales de los Países Miembros de la Comunidad Andina deberán tener en cuenta al momento de resolver los recursos de anulación de laudos:

i. Cuando el recurso de anulación esté sustentado por la falta de solicitud obligatoria de interpretación prejudicial del árbitro: la instancia nacional declarará nulo el laudo y elegirá entre la devolución del asunto al árbitro que debió solicitar la interpretación prejudicial para que subsane su omisión y emita un 
nuevo laudo, o de lo contrario si el ordenamiento interno no dispone la devolución, esta instancia nacional tendrá que emitir una resolución sustitutiva donde solicite de forma obligatoria la interpretación prejudicial.

ii. Cuando el recurso de anulación no sustente la falta de la solicitud de interpretación prejudicial del árbitro pero sí se refiera a la interpretación de normas comunitarias o deban aplicarlas: la instancia nacional también declarará nulo el laudo y seguirá con las mismas pautas indicadas en el punto (i) referentes a la devolución del asunto o la resolución sustitutiva.

En cuanto al análisis de la jurisprudencia del Tribunal de Justicia de la Unión Europea, este no reconoce a un tribunal arbitral de origen privado como órgano jurisdiccional; no utiliza como fundamento las similitudes entre la actividad arbitral y la jurisdiccional. Dicha postura lo estableció desde la sentencia del Tribunal de Justicia del 23 de marzo de 1982 sobre el «Asunto 102/81: Nordsee Deutsche Hochseefischerei GmbH contra Reederei Mond Hochseefischerei Nordstern AG \& Co. KG y Reederei Friedrich Busse Hochseefischerei Nordstern AG \& Co. KG», numerales 10 al 13. De esta línea argumentativa, se deriva la decisión del Tribunal de Justicia de la Unión Europea para establecer criterios por los cuales ciertos tribunales arbitrales puedan ser considerados como órganos jurisdiccionales competentes para reenviar la cuestión prejudicial. Con el Auto del Tribunal de Justicia (Sala Octava) del 13 de febrero de 2014 sobre el «Asunto C-555/13: Merck Canada Inc. contra Accord Healthcare Ltd y otros. Petición de decisión prejudicial planteada por el Tribunal Arbitral necessário. Procedimiento prejudicial - Concepto de «órgano jurisdiccional nacional» en el sentido del artículo 267 TFUE - Tribunal Arbitral necessário - Admisibilidad - Reglamento (CE) no 469/2009 - Artículo 13 - Certificado complementario de protección para los medicamentos - Duración de un certificado - Período máximo de exclusividad», numerales 16 al 25, se estableció que un tribunal arbitral podrá ser calificado como órgano jurisdiccional si también posee los criterios de definición que el Tribunal de Justicia reiteradamente señala en su jurisprudencia: el origen legal del órgano, su permanencia, el carácter obligatorio de su jurisdicción, el carácter contradictorio del procedimiento, la aplicación por parte del órgano de normas jurídicas y su independencia.

Recientemente, derivada de una disputa entre la compañía neerlandesa Achmea BV y Eslovaquia, el Tribunal de Justicia de la Unión Europea evaluó una solicitud de cuestión prejudicial presentada por el Bundesgerichtshof (Tribunal Supremo de lo Civil y Penal alemán), órgano jurisdiccional encargado de resolver de manera definitiva la anulación del laudo arbitral, la misma que favorece a Achmea BV desde el 7 de diciembre de 2012. Una de las principales conclusiones a la que llega el Tribunal de Justicia es que un tribunal arbitral creado por un acuerdo internacional como el «Tratado para el Fomento y la Protección Recíprocos de las Inversiones entre 
el Reino de los Países Bajos y la República Federal Checa y Eslovaca» de 1991 (es decir que tenga su origen por su disposición de un Tratado Bilateral de Inversiones entre Estados Miembros de la Unión Europea suscrito con anterioridad a sus respectivas Adhesiones), no constituye elemento del sistema judicial de los Estados Miembros, y tampoco es común a todos ellos.

Por consiguiente, un tribunal arbitral internacional que se instale bajo las mismas circunstancias, no podrá ser visto por el Tribunal de Justicia de la Unión Europea como órgano jurisdiccional legitimado para solicitarle la cuestión prejudicial. Tal decisión se encuentra sustentada en los numerales 44 al 49 de la sentencia del Tribunal de Justicia (Gran Sala) del 6 de marzo de 2018 sobre el «Asunto 284/16: Procedimiento prejudicial - Tratado Bilateral de Inversión celebrado en 1991 entre el Reino de los Países Bajos y la República Federal Checa y Eslovaca y que sigue siendo aplicable entre el Reino de los Países Bajos y la República Eslovaca— Disposición que permite a un inversor de una Parte contratante dirigirse a un tribunal arbitral en caso de litigio con la otra Parte contratante - Compatibilidad con los artículos 18 TFUE, 267 TFUE y 344 TFUE- Concepto de «órgano jurisdiccional» - Autonomía del Derecho de la Unión».

Asimismo, en los numerales 57 y 58 de la sentencia se explica las consecuencias de que un tribunal arbitral internacional, constituido conforme a un tratado bilateral de inversiones entre Estados Miembros de la Unión Europea, laude en base al Derecho vigente en el Estado Miembro contratante afectado. Una de ellas refiere sobre el Derecho de la Unión Europea al ser parte del ordenamiento jurídico interno del Estado Miembro que resulte afectado frente al incumplimiento de su contraparte. El Tribunal de Justicia de la Unión Europea llega a la conclusión de que «el Derecho de la Unión Europea podría ser interpretado y aplicado» en caso de que un tribunal arbitral internacional (de ese tipo) se constituya. Según el Tribunal de Justicia, lo último significa «un peligro para la preservación del carácter propio del Derecho de la Unión Europea, dado que se estaría vulnerando su autonomía, la misma que está establecida por los Tratados y que se encuentra garantizado por el procedimiento de remisión prejudicial del Artículo 267 del Tratado de Funcionamiento de la Unión Europea».

De otro lado, al igual que en el caso del funcionario administrativo con función jurisdiccional, observamos que la interpretación del término juez nacional del Tribunal Andino continua siendo inadecuada por considerar al árbitro en derecho también como un operador judicial. Es evidente que el árbitro no posee la facultad coercitiva del juez, no cuenta con un nombramiento estatal, no es impuesto a las partes, su rol no es permanente, no es reemplazable y no puede efectuar por sí mismo ninguna medida cautelar. Además, aunque los laudos arbitrales producen efectos similares de cosa juzgada, estos no pueden ser ejecutados por los propios árbitros; para cumplir con sus decisiones 
también se necesita de la asistencia judicial. Finalmente, cabe señalar que lograr la unidad y la autonomía del Derecho Comunitario Andino no justifica que el órgano jurisdiccional comunitario andino realice interpretaciones simples, más aún cuando el Tribunal Andino es considerado, en términos del número de las sentencias, como el tercer tribunal internacional más activo a nivel mundial (Alter y Helfer, 2017, p. 262).

\section{Conclusiones}

Culminado nuestro análisis de la labor interpretativa que el Tribunal Andino realiza sobre el término juez nacional, que es quien solicita la interpretación prejudicial, podemos arribar a las siguientes conclusiones:

Aunque el Tribunal Andino precisa que no dejará de lado el resto de métodos de interpretación, el órgano jurisdiccional de la Comunidad Andina ha privilegiado reiteradamente en sus resoluciones a la interpretación teleológica o funcional porque según su criterio analítico, esta se adapta mejor para cumplir con su obligación de aplicar uniformemente el ordenamiento andino.

En ninguna de las sentencias analizadas sobre el término juez nacional del Artículo 33 del Tratado que crea el Tribunal de Justicia de la Comunidad Andina, se hace mención alguna a las reglas de interpretación de la Convención de Viena sobre el Derecho de los Tratados. Es más, del análisis de tales sentencias tampoco se aprecia que indirectamente haya seguido tales reglas, y no detalla cuáles fueron los pasos de su proceso interpretativo en cada solicitud que le fue planteada.

Si bien el Tribunal Andino resalta el principio de cooperación y colaboración con el juez nacional para garantizar la correcta interpretación del ordenamiento andino y su aplicación uniforme en todos los Países Miembros de la Comunidad Andina, su afán por cumplir con tales preceptos ha ocasionado, a nuestro parecer, una inapropiada identificación del legítimo operador nacional, ya que este tribunal de justicia entiende que «juez nacional» también es un funcionario administrativo con función jurisdiccional y un árbitro.

A diferencia de la postura del Tribunal de Justicia de la Unión Europea, el Tribunal Andino considera que todos los tribunales arbitrales de los Países Miembros de la Comunidad Andina, aunque hayan sido constituidos por la voluntad de las partes, es decir, que no tengan un origen legal nacional y mucho menos sean permanentes, tienen la obligación de solicitarle la interpretación prejudicial cuando en sus procedimientos arbitrales se ventilen normas comunitarias andinas. 


\section{Referencias bibliográficas}

Alter, K. J. y Helfer, L. R. (2017). Transplanting International Courts. The Law and Politics of the Andean Tribunal of Justice. Oxford: Casa Editorial de la Universidad de Oxford. https://doi.org/10.1093/acprof:oso/9780199680788.001.0001

Cannizzaro, E. (ed.) (2011). The Law of Treaties beyond the Vienna Convention. Oxford: Casa Editorial de la Universidad de Oxford.

Comunidad Andina (s.f.). Acuerdo de Integración Subregional (Acuerdo de Cartagena) de 1969.

Comunidad Andina (1996). Tratado de Creación del Tribunal de Justicia de la Comunidad Andina.

Consejo Andino de Ministros de Relaciones Exteriores (2001). Estatuto del Tribunal de Justicia de la Comunidad Andina. Decisión 500.

Del Valle Gálvez, J. A. (1995). La interpretación de Tratados internacionales por el TJCE (comentario a las sentencias de 1 de junio de 1993, Metalsa y Eurim-pharm). Revista de Instituciones Europeas, 22(3), 861-886.

Gándara, P. y Bodenstein, T. (2006). La Política Económica de Integración Regional en Europa y América Latina. En J. M. Beneyto (dir.) y P. Argerey (coord.), Europa y América Latina. El otro diálogo transatlántico (pp. 265-294). Madrid: Editorial Biblioteca Nueva.

Kolb, R. (2016). The Law of Treaties: an Introduction. Cheltenham: Editor Edward Elgar. https://doi.org/10.4337/9781785360152

Levi, M. (2006). La Unión Europea y la Comunidad Andina: relaciones entre los procesos de Integración. En J.M. Beneyto (dir.) y P. Argerey (coord.), Europa y América Latina. El otro diálogo transatlántico (pp. 113-136). Madrid: Editorial Biblioteca Nueva.

Linderfalk, U. (2010). On the Interpretation of Treaties: the Modern International Law as expressed in the 1969 Vienna Convention on the Law of Treaties. Dordrecht: Editorial Springer.

Novak, F. (2003). La Comunidad Andina y su Ordenamiento Jurídico. En IDEI (eds.), Derecho Comunitario Andino (pp. 57-100). Lima: PUCP - Fondo Editorial.

Novak, F. (2013). Los Criterios para la interpretación de los Tratados. En: Revista de Derecho THEMIS, 63, 71-88.

Orakhelashvili, A. (2008). The Interpretation of Acts and Rules in Public International Law. Oxford, Nueva York: Casa Editorial de la Universidad de Oxford. https://doi. org/10.1093/acprof:oso/9780199546220.001.0001

Organización de Naciones Unidas (1969). Convención de Viena sobre el Derecho de los Tratados.

Peńa, F. y Cárdenas, E. J. (1968). Los acuerdos subregionales y el Tratado de Montevideo. Revista de Derecho de la Integración, 2, 10-19.

Perotti, A. D. (2002). Algunas consideraciones sobre la interpretación prejudicial obligatoria en el derecho andino. Revista Díkaion, 16(11), 127-153.

Quindimil López, J. A. (2006). Instituciones y Derecho de la Comunidad Andina. Valencia: Tirant lo Blanch. 
Tribunal de Justicia de la Comunidad Andina (1987). Proceso N 1-IP-87, Recuperado de http://intranet.comunidadandina.org/documentos/Procesos/1-IP-1987.doc

Tribunal de Justicia de la Comunidad Andina (1989). Proceso $N^{\circ} 1-89$. Recuperado de http://www.comunidadandina.org/DocOficialesFiles/Procesos/Providencia\%20 (25-04-89)\%2043.doc

Tribunal de Justicia de la Comunidad Andina (1995). Proceso 04-IP-94, Recuperado de intranet.comunidadandina.org/Documentos/Procesos/4-IP-94.doc

Tribunal de Justicia de la Comunidad Andina (2007). Proceso N 14-IP-2007. Recuperado de http://intranet.comunidadandina.org/documentos/Procesos/14-IP-2007.doc

Tribunal de Justicia de la Comunidad Andina (2007). Proceso N 141-IP-2007. Recuperado de http://intranet.comunidadandina.org/documentos/Procesos/141-IP-2007.doc

Tribunal de Justicia de la Comunidad Andina (2010). Proceso No 03-AI-2010. Recuperado de http://intranet.comunidadandina.org/documentos/Procesos/3-AI-2010.doc

Tribunal de Justicia de la Comunidad Andina (2012). Proceso N 57-IP-2012. Recuperado de http://intranet.comunidadandina.org/documentos/Procesos/57-IP-2012.doc

Tribunal de Justicia de la Comunidad Andina (2014). Proceso N 121-IP-2014. Recuperado de http://intranet.comunidadandina.org/documentos/Procesos/121-IP-2014.doc

Tribunal de Justicia de la Unión Europea (1966). Sentencia del Tribunal de Justicia, Asunto 61/65. Recuperado de http://curia.europa.eu/juris/showPdf.jsf?text=\&docid=87458 \&pageIndex $=0$ \&doclang $=E S \&$ mode $=1$ st $\&$ dir $=\& o c c=$ first $\&$ part $=1 \&$ cid $=1103276$

Tribunal de Justicia de la Unión Europea (1982). Sentencia del Tribunal de Justicia, Asunto 102/81. Recuperado de http://curia.europa.eu/juris/showPdf.jsf?text=\&docid=9140 2\&pageIndex=0\&doclang=ES\&mode=lst \&dir $=\&$ occ $=$ first $\&$ part $=1 \&$ cid $=1104761$

Tribunal de Justicia de la Unión Europea (1986). Auto del Tribunal de Justicia, Asunto 318/85. Recuperado de http://curia.europa.eu/juris/showPdf.jsf?text=\&docid=9418 9\&pageIndex $=0$ \&doclang $=$ ES\& $\&$ mode $=1$ st $\&$ dir $=\&$ occ $=$ first $\&$ part $=1 \&$ cid $=3126855$

Tribunal de Justicia de la Unión Europea (1987). Sentencia del Tribunal de Justicia (Sala Quinta), Asunto 14/86. Recuperado de http://curia.europa.eu/juris/showPdf.jsf?tex $\mathrm{t}=\&$ docid $=94575 \&$ pageIndex $=0$ \&doclang $=\mathrm{ES} \&$ mode $=1$ st $\&$ dir $=\& o c c=$ first $\&$ part $=1$ \&cid $=3127152$

Tribunal de Justicia de la Unión Europea (1990). Sentencia del Tribunal de Justicia, Asuntos acumulados C-100/89 y C-101/89. Recuperado de http://curia.europa.eu/juris/ showPdf.jsf?text $=\&$ docid $=96672 \&$ pageIndex $=0 \&$ doclang $=E S \&$ mode $=1$ st $\&$ dir $=\&$ oc c=first \&part=1\&cid=3127406

Tribunal de Justicia de la Unión Europea (1997). Sentencia del Tribunal de Justicia, Asunto C-337/95. Recuperado de http://curia.europa.eu/juris/document/document.jsf?text $=\&$ docid $=43440 \&$ pageIndex $=0 \&$ doclang $=E S \&$ mode $=1$ st $\&$ dir $=\&$ occ $=$ first $\&$ part $=1$ \&cid $=3127514$

Tribunal de Justicia de la Unión Europea (1999). Auto del Tribunal de Justicia, Asunto C-192/98. Recuperado de http://curia.europa.eu/juris/showPdf.jsf?text=\&docid=484 84\&pageIndex $=0 \&$ doclang $=\mathrm{ES} \&$ mode $=1 \mathrm{st} \& \mathrm{dir}=\&$ occ $=$ first $\&$ part $=1 \& \mathrm{cid}=3127610$ 
Tribunal de Justicia de la Unión Europea (2014). Auto del Tribunal de Justicia (Sala Octava), Asunto C-555/13. Recuperado de http://curia.europa.eu/juris/document/document. jsf?text $=\&$ docid $=148021 \&$ pageIndex $=0 \&$ doclang $=$ es $\&$ mode $=1$ st $\&$ dir $=\&$ occ $=$ first $\&$ part $=1 \& \operatorname{cid}=481874$

Tribunal de Justicia de la Unión Europea (2018). Sentencia del Tribunal de Justicia (Gran Sala), Asunto 284/16. Recuperado de http://curia.europa.eu/juris/document/document.jsf?text $=\&$ docid $=199968 \&$ doclang $=\mathrm{ES}$

Villiger, M.E. (2009). Commentary on the 1969 Vienna Convention on the Law of Treaties. Leiden, Boston: Editorial Martinus Nijhoff. https://doi.org/10.1163/ ej.9789004168046.i-1058

Recibido: 29 de marzo de 2019

Aprobado: 7 de agosto de 2019 